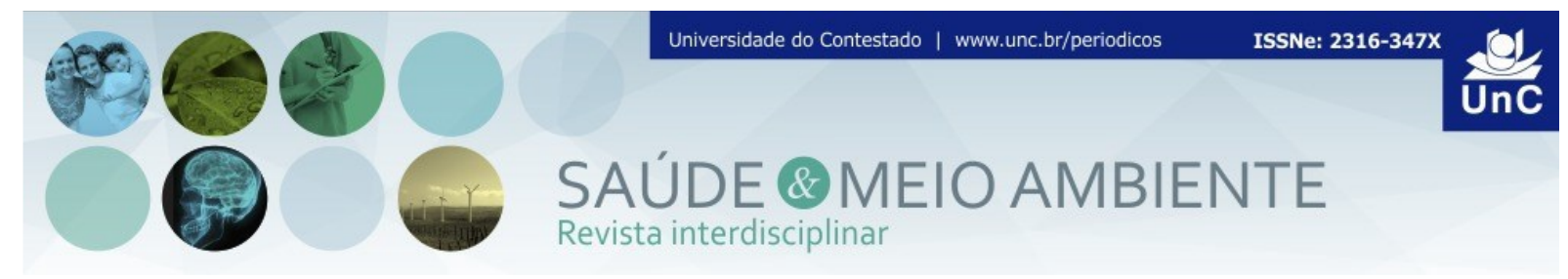

\title{
AVALIAÇÃO DO PERFIL DOS PACIENTES QUE FREQUENTAM OS LABORATÓRIOS DE ESTÉTICA EM RELAÇÃO AO USO DE NUTRICOSMÉTICOS
}

\section{ASSESSMENT OF THE PROFILE OF PATIENTS ATTENDING AT AESTHETIC LABORATORIES ACCORDING USE NUTRICOSMETICS}

\author{
Amanda Vieira de Cândido ${ }^{1}$ \\ Letícia Formolo ${ }^{2}$ \\ Bruna Bellincanta Nicoletto ${ }^{3}$
}

\begin{abstract}
RESUMO
Introdução: As pessoas estão se preocupando cada vez mais com a sua imagem pessoal, com isso o mercado de cosméticos continua com um crescimento anual significativo. Os nutricosméticos estão cada vez mais inseridos na vida das pessoas. Objetivo: Avaliar o perfil de pacientes que frequentam o Laboratório de Estética e Cosmética da Universidade de Caxias do Sul, quanto ao uso de nutricosméticos. Métodos: Trata-se de um estudo transversal, incluindo todos os pacientes que frequentaram o laboratório de abril a julho de 2019. Os dados foram coletados em prontuários preenchidos durante os atendimentos. Foram coletados dados sociodemográficos e questões de saúde do paciente, além de questões relacionadas ao uso de nutricosméticos. Resultados: Foram incluídos 95 prontuários na análise de dados do estudo. Da amostra total de indivíduos, 49,5\% utilizavam nutricosméticos. Os produtos citados com mais frequência foram vitamina D $(22,6 \%)$, colágeno $(14 \%)$, ômega $3(9,7 \%)$, multivitamínico $(9,7 \%)$ e vitamina C (8,6\%). Entre os usuários de nutricosméticos, houve maior frequência de mulheres (100\% vs $87 \% ; p=0,012)$, e de maior idade, com diferença significativa em comparação aos não usuários $(31,76 \pm$ 13,42 anos vs 41,09 $\pm 17,74$ anos). Conclusão: O perfil de indivíduos que frequentam os laboratórios de Estética e Cosmética da Universidade de Caxias do Sul quanto à utilização de nutricosméticos são principalmente mulheres de média idade que utilizam vitamina $\mathrm{D}$ e colágeno.
\end{abstract}

Palavras-chave: Suplementos Nutricionais. Cosmecêuticos. Cosméticos.

\footnotetext{
${ }^{1}$ Acadêmica do Curso Tecnológico de Estética e Cosmética na Universidade de Caxias do Sul. Rio Grande do Sul. Brasil. E-mail: avcandido@ucs.br

${ }^{2}$ Acadêmica do Curso Tecnológico de Estética e Cosmética na Universidade de Caxias do Sul. Rio Grande do Sul. Brasil. E-mail: Iformolo@ucs.br

${ }^{3}$ Docente da Área do Conhecimento de Ciências da Vida, na Universidade de Caxias do Sul. Nutricionista. Mestre em Ciências Médicas. Doutora em Endocrinologia. Universidade de Caxias do Sul. Rio Grande do Sul. Brasil. E-mail: bbngehrke@ucs.br
} 


\begin{abstract}
Introduction: As people are increasingly worrying about their personal image, the cosmetic market continues to grow significantly annually. Nutricosmetics are increasingly embedded in people's lives. Objective: To analyze the profile of patients attending at the Aesthetics and Cosmetics Laboratory of the University of Caxias do Sul, regarding the use of nutricosmetics. Methods: This was a cross-sectional study, including all patients attending the laboratory in April to July of 2019. Data were collected from completed medical records. Sociodemographic data and patient health issues were collected, as well as questions related to the use of nutricosmetics. Result: 95 medical records were included in the study data analysis. Of the total sample of individuals, $49.5 \%$ use nutricosmetics. The most frequently cited products were vitamin D (22.6\%), collagen (14\%), omega $3(9.7 \%)$, multivitamin $(9.7 \%)$ and vitamin C (8.6\%). Among nutricosmetic users, there was a higher frequency of women $(100 \%$ vs $87 \% ; p=0.012)$, and older age, with a significant difference compared to non-users (31.76 \pm 13.42 years vs $41.09 \pm 17,74$ years old). Conclusion: The profile of individuals who attend at the Aesthetics and Cosmetics laboratories of the University of Caxias do Sul regarding the use of nutricosmetics is mainly middle-aged women who use vitamin $\mathrm{D}$ and collagen.
\end{abstract}

Keywords: Dietary Supplements. Cosmeceuticals. Cosmetics.

\title{
INTRODUÇÃO
}

Desde antigamente as pessoas se preocupam com sua imagem. A beleza continua sendo desejada pelos indivíduos nos dias de hoje, principalmente devido aos padrões estabelecidos pela sociedade. A construção da feminilidade tem estado diretamente ligada à noção de beleza. Quanto mais bonita uma mulher, mais feminina é considerada ${ }^{1}$.

Atualmente a exposição do corpo está maior, logo, as mulheres desejam um corpo perfeito, sem afecções vistas como algo negativo para sua feminilidade. Visto a invasão da mídia neste contexto, as mulheres acabam procurando maneiras de se livrar do excesso de adiposidade em lugares como o abdômen, além de outros transtornos que as incomodam². Pensando em maneiras de buscar a beleza, as pessoas começaram a adquirir estilos de vida alternativos. Além da alimentação, um novo conceito de produto tem ganho destaque, os nutricosméticos ${ }^{3}$.

O cuidar do organismo "de dentro para fora" surge da hipótese de que cremes ou outras soluções tópicas não resolvem completamente o problema ${ }^{4}$. Especialistas da indústria cosmética, aliados à indústria de alimentos, pesquisam inúmeras combinações de nutrientes e bioativos com princípios de promover firmeza e hidratação da pele, combate aos radicais livres, fortalecimento de unhas e cabelos, estímulo do metabolismo, fotoproteção e outros relacionados ao bem-estar. $\mathrm{O}$ ácido ascórbico, popularmente conhecido como vitamina $\mathrm{C}$ é um poderoso antioxidante nutricosmético, além do colágeno, ômega 3 , entre outros ${ }^{5}$. 
De acordo com a Agência Nacional de Vigilância Sanitária (ANVISA), o termo nutricosméticos não está normatizado até o momento. A ANVISA enquadra os produtos nutricosméticos na categoria de alimentos funcionais, porque produzem efeitos metabólicos ou fisiológicos por meio da atuação de um nutriente na manutenção do organismo ${ }^{23,24}$. Assim, são considerados suplementos nutricionais baseados em compostos bioativos que possuem atividade antioxidante, antiinflamatória, antiglicante, desglicante, entre outras ${ }^{4}$.

Dentre o público que mais procura os nutricosméticos está o sexo feminino ${ }^{6}$. Decorrente dessa mudança no mercado da beleza, o objetivo do trabalho foi avaliar o perfil de pacientes que frequentam o Laboratório de Estética da Universidade de Caxias do Sul, quanto ao uso de nutricosméticos.

\section{MÉTODOS}

\section{DESENHO DO ESTUDO E POPULAÇÃO DE PESQUISA}

Trata-se de um estudo transversal, incluindo todos os pacientes que frequentaram o laboratório de Estética e Cosmética da Universidade de Caxias do Sul no período de abril a julho de 2019. Os dados foram coletados em prontuários preenchidos durante os atendimentos de rotina. Foram excluídos prontuários incompletos quanto ao uso de nutricosméticos.

O protocolo do presente estudo foi aprovado pelo Comitê de Ética e Pesquisa da Universidade de Caxias do Sul, RS, Brasil, sob $n^{\circ}$ 17931419.7.0000.5341. O projeto de pesquisa foi elaborado em consonância com as Diretrizes e Normas Regulamentadoras de Pesquisas Envolvendo Seres Humanos, aprovadas pelo Conselho Nacional de Saúde, resolução número 466, de 2012. Os pesquisadores assinaram o termo de compromisso para utilização de dados.

\section{COLETA DE DADOS}

Todos os dados foram coletados de prontuários com o registro conforme a realização dos atendimentos de rotina. Estavam disponíveis nos prontuários questões referentes a dados sociodemográficos e questões de saúde do paciente. As variáveis extraídas do prontuário foram: idade, gênero, etnia, faixa salarial (em salários mínimos), escolaridade, consumo de bebidas alcoólicas, tabagismo, nível de estresse (classificado em baixo, médio e alto), ingestão de líquidos (1 Litros, 1,5 Litros ou 2 Litros), adoção de alimentação saudável, prática de atividade física e funcionamento intestinal (regular ou irregular), além de informações referentes às doenças, como: 
anemia, hipertensão, diabetes mellitus ou disfunção em tireoide. Além disso, foram coletadas dos prontuários questões relacionadas ao uso de nutricosméticos, incluindo quais nutricosméticos, se houve orientação de algum profissional da saúde ou se teve indicação de outras fontes e se observou alguma mudança depois do tratamento.

Todas as variáveis foram referidas pelo próprio paciente durante o atendimento de rotina e registradas em prontuário, sendo este posteriormente consultado para coleta de dados da presente pesquisa.

\section{ANÁLISE ESTATÍSTICA}

Os dados foram analisados através do programa Statistical Package for Social Sciences, versão 20.0 (SPSS Inc, Chicago, IL). O teste de Shapiro Wilk foi utilizado para verificação da normalidade das variáveis contínuas. As variáveis com distribuição normal foram apresentadas como média \pm desvio padrão. As variáveis categóricas foram apresentadas em frequências absolutas e percentuais. Os pacientes que utilizavam e não utilizavam nutricosméticos foram comparados de acordo com variáveis sociodemográficas, clínicas e de estilo de vida, utilizando os testes $t$ de Student, Mann-Whitney ou Qui-quadrado, conforme apropriado. Para determinação da significância estatística foi considerado o valor de $p<0,05$.

\section{RESULTADOS}

No período de abril a julho de 2019, foram atendidos 179 pacientes no Laboratório de Estética e Cosmética da Universidade de Caxias do Sul. Todos os prontuários foram acessados para avaliação dos critérios de inclusão. Destes, foram excluídos 84 prontuários por estarem incompletos quanto aos itens sobre nutricosméticos. Assim, 95 prontuários foram incluídos na análise de dados do estudo.

A idade média dos pacientes foi de $36,5 \pm 16,4$ anos, sendo a maioria do sexo feminino $(n=87 ; 93,5 \%)$. Da amostra total de indivíduos, 47 (49,5\%) utilizavam nutricosméticos. Os produtos citados com mais frequência foram: vitamina $D(n=21$; $22,6 \%)$, colágeno $(n=13 ; 14 \%)$, ômega $3(n=9 ; 9,7 \%)$, multivitamínico $(n=9 ; 9,7 \%)$ e vitamina $C(n=8 ; 8,6 \%)$. Dos 47 pacientes que utilizavam nutricosméticos, $47,83 \%$ $(n=22)$ tiveram indicação através de médicos, $28,26 \%(n=13)$ através de amigos, familiares ou colegas de trabalho e $15,22 \%(n=7)$ através de internet ou televisão e apenas $4,35 \% \quad(n=2)$ de nutricionistas. Notaram mudanças após o uso de nutricosméticos $72,09 \%(\mathrm{n}=31)$ dos indivíduos.

A tabela 1 apresenta as características sociodemográficas e de estilo de vida de acordo com o uso de nutricosméticos. Entre os indivíduos que utilizavam nutricosméticos, a frequência de mulheres foi maior ( $100 \%$ vs $87 \%$; $p=0,012)$, assim 
como a idade $(41,09 \pm 17,74$ anos vs $31,76 \pm 13,42$ anos; $p=0,012)$, em comparação aos que não utilizavam nutricosméticos. Além disso, os indivíduos apresentaram diferença significativa quanto a presença de hipertensão, sendo 5 casos $(11,4 \%)$ no grupo de usuários de nutricosméticos e nenhum no grupo de não usuários $(p=0,026)$. As demais variáveis não foram diferentes entre os grupos.

De maneira geral, a maioria da população era solteira, com ensino superior completo e nível de estresse médio. Ambos os grupos tiveram índice baixo de usuários de tabagismo e o consumo de bebida alcoólica esteve presente em 39,2\% da amostra total. Entre os dois grupos, 52 indivíduos praticavam atividade física e 58 consideravam ter alimentação balanceada. Da população total, 91,1\% ingere 1 litro de água por dia e 71 indivíduos possuem intestino regular. O motivo da procura pelos serviços nos laboratórios de Estética e Cosmética, foram em $56 \%$ dos casos devido às manchas (Tabela 1).

Tabela 1 - Perfil da amostra de acordo com o uso de nutricosméticos.

\begin{tabular}{|c|c|c|c|}
\hline Variáveis & $\begin{array}{c}\text { Usuários de } \\
\text { nutricosméticos } \\
(n=47)\end{array}$ & $\begin{array}{c}\text { Não usuários de } \\
\text { nutricosméticos } \\
(n=48)\end{array}$ & P valor \\
\hline Sexo feminino, $n(\%)$ & $47(100)$ & $40(87,0)$ & 0,012 \\
\hline Idade, anos & $41,09 \pm 17,74$ & $31,76 \pm 13,42$ & 0,006 \\
\hline Estado civil, $n(\%)$ & & & 0,355 \\
\hline Solteiro & $28(56,9)$ & $30(66,7)$ & \\
\hline Casado & $13(27,7)$ & $14(31,1)$ & \\
\hline Divorciado & $3(6,4)$ & $0(0)$ & \\
\hline Viúvo & $2(4,3)$ & $1(2,2)$ & \\
\hline União estável & $1(2,1)$ & $0(0)$ & \\
\hline Escolaridade, $\mathrm{n}(\%)$ & & & 0,512 \\
\hline Fundamental Incompleto & $2(4,8)$ & $2(4,9)$ & \\
\hline Fundamental Completo & $5(11,9)$ & $3(7,3)$ & \\
\hline Médio Incompleto & $0(0)$ & $4(9,8)$ & \\
\hline Médio Completo & $10(23,8)$ & $7(17,1)$ & \\
\hline Técnico & $0(0)$ & $1(2,4)$ & \\
\hline Superior Incompleto & $8(19,0)$ & $9(22,0$ & \\
\hline Superior Completo & $13(31,0)$ & $11(26,8)$ & \\
\hline Pós Graduação/ Mestrado & $4(9,5)$ & $4(9,8)$ & \\
\hline Salários mínimos, número & $2,20 \pm 1,41$ & $1,82 \pm 1,72$ & 0,271 \\
\hline$\overline{\text { Nível de estresse, } \mathrm{n}(\%)}$ & & & 0,649 \\
\hline Alto & $8(17,4)$ & $8(17,4)$ & \\
\hline Médio & $20(43,5)$ & $24(52,2)$ & \\
\hline Baixo & $18(39,1)$ & $14(30,4)$ & \\
\hline Consumo de bebida alcoólica, n (\%) & $7(15,9)$ & $10(23,3)$ & 0,429 \\
\hline Tabagismo, n (\%) & $2(4,3)$ & $4(8,7)$ & 0,435 \\
\hline Alimentação balanceada n (\%) & $28(62,2)$ & $30(68,2)$ & 0,557 \\
\hline Pratica atividade física, n (\%) & $28(60,9)$ & $24(53,3)$ & 0,470 \\
\hline Consumo de líquido, n (\%) & & & 0,380 \\
\hline
\end{tabular}


Avaliação do perfil dos pacientes que frequentam os laboratórios de estética em relação ao uso de nutricosméticos

\begin{tabular}{lccc}
\hline 1 Litro & $19(42,2)$ & $22(48,9)$ & \\
\hline 1,5 Litros & $17(37,8)$ & $11(24,4)$ & \\
\hline 2 Litros & $9(20,0)$ & $12(26,7)$ & \\
\hline Intestino regular, $\mathrm{n}(\%)$ & $36(81,8)$ & $35(81,4)$ & 0,960 \\
\hline Doenças da tireóide & $10(21,3)$ & $5(11,1)$ & 0,261 \\
\hline Anemia & $1(2,2)$ & $1(2,2)$ & 0,999 \\
\hline Hipertensão & $5(11,4)$ & $0(0)$ & 0,026 \\
\hline Diabetes mellitus & $2(4,3)$ & $1(2,2)$ & 0,999 \\
\hline Motivo de consulta, $\mathrm{n}(\%)$ & & & \\
\hline Manchas & $11(23,4)$ & $15(32,6)$ & 0,325 \\
\hline Espinhas & $5(10,6)$ & $10(21,7)$ & 0,148 \\
\hline Cravos & $7(14,9)$ & $10(21,7)$ & 0,396 \\
\hline Dores no corpo & $4(8,5)$ & $2(4,3)$ & 0,677 \\
\hline Oleosidade & $6(12,8)$ & $6(13,0)$ & 0,968 \\
\hline Rugas & $4(8,5)$ & $2(4,3)$ & 0,677 \\
\hline Flacidez & $9(19,1)$ & $4(8,7)$ & 0,148 \\
\hline Olheiras & $0(0)$ & $1(2,2)$ & 0,495 \\
\hline Pele seca & $4(8,5)$ & $2(4,3)$ & 0,677 \\
\hline Rosácea & $1(2,1)$ & $1(2,2)$ & 0,988 \\
\hline Fibro Edema Gelóide & $1(2,1)$ & $2(4,3)$ & 0,617 \\
\hline Adiposidade & $6(12,8)$ & $6(13,0)$ & 0,968 \\
\hline
\end{tabular}

Fonte: Dados da Pesquisa (2019).

\section{DISCUSSÃO}

O presente estudo identificou que metade da população utiliza nutricosméticos, sendo o mais prevalente entre os indivíduos a vitamina $D$, com indicação de profissional da saúde em $52,18 \%$ dos casos. Os usuários de nutricosméticos apresentaram maior idade, além de maior frequência de indivíduos do sexo feminino e hipertensos.

Percebe-se que a preocupação com a beleza é alvo das atenções de toda humanidade, não se limitando a classes sociais e faixas etárias, tornando a sociedade a principal responsável pela formação de opinião. Porém, observa-se que o sexo feminino é o grupo que mais procura realizar mudanças na sua aparência ${ }^{7}$. Da mesma forma, neste estudo a procura por nutricosméticos foi maior pelo público feminino no Laboratório de Estética e Cosmética da Universidade de Caxias do Sul.

A mídia e a sociedade estão envolvidas com a busca pela beleza, impondo rótulos que pessoas se submetem a seguir, para que não sejam excluídas do ambiente ao qual pertencem ${ }^{7}$. Em consequência disso, aumenta a busca por alterar $\mathrm{o}$ corpo com um todo ${ }^{8}$. Em 2013, foi conduzida uma pesquisa pela American Society of Plastic Surgeons [ASPS] ${ }^{21}$ (Sociedade Americana de Cirurgiões Plásticos), divulgada no site da Sociedade Brasileira de Cirurgias Plásticas), que apontou o Brasil como o primeiro colocado no ranking mundial de cirurgias plásticas realizadas, ficando à frente dos EUA com 1.491,721 do total de procedimentos realizados, mais um dado que 
confirma a procura exacerbada das mulheres pela beleza ${ }^{22}$. Assim, os nutricosméticos têm sido procurados pela população, em especial mulheres, com o objetivo de atender às expectativas na busca pela beleza, por vezes associando o uso a tratamentos tópicos e diminuindo o número de tratamentos invasivos ${ }^{4,20}$.

Além do sexo feminino, indivíduos com maior idade estiveram entre os usuários de nutricométicos no presente estudo. É provável que mulheres com mais idade demonstrem maior preocupação com os sinais do envelhecimento e por esse motivo utilizem os nutricosméticos em maior proporção. Em outro estudo, analisando o uso de ômega 3, a idade média dos usuários foi 23,7 anos, no entanto a maioria dos participantes eram homens ${ }^{9}$. Já em outra pesquisa, com um grupo de participantes que fizeram uso de colágeno, a idade média foi de 55,6 anos ${ }^{10}$. A variação da faixa etária do público que busca nutricosméticos está atrelada, possivelmente, ao tipo de produto e objetivo do público.

Os hábitos alimentares e a prática de atividade física não estiveram diretamente relacionados com a procura por nutricosméticos por parte dos indivíduos deste estudo. Estes resultados diferem dos achados de Striegel e seus colaboradores $(2006)^{12}$, que referem em um estudo realizado com atletas participantes do Campeonato Mundial de Atletismo Internacional em 2004 que 60,5\% de todos os participantes relataram o uso real de suplementos nutricionais. No entanto não foram encontradas diferenças significativas entre usuários de suplementos nutricionais e não usuários em relação às informações básicas de desempenho, ou seja, ainda não existem comprovações específicas quanto às mudanças de desempenho e saúde para os usuários de nutricosméticos, quando comparados com os que não fazem uso $^{12}$.

Existem vários estudos $9,19,20$ avaliando a eficácia dos nutricosméticos, demonstrando seu valor benéfico para o organismo. Porém ainda são necessárias mais pesquisas com diferentes populações. Salienta-se que estes produtos são vendidos de forma livre em farmácias, supermercados e lojas de produtos de beleza, tendo livre acesso de circulação à população em geral.

A vitamina $D$ foi o nutricosmético mais utilizado pela população deste estudo. Neste estudo, $22,6 \%$ dos pacientes estavam em uso desse nutricosmético. Sabe-se que a vitamina $\mathrm{D}$ tem ação no metabolismo do cálcio, na formação óssea, no sistema imunológico, entre outros. Além disso, a deficiência dessa vitamina está relacionada com várias doenças autoimunes, como diabetes, esclerose múltipla e artrite reumatoide ${ }^{11}$. Esse suplemento pode desempenhar um papel importante também no combate a doenças cardiovasculares ${ }^{13}$.

Outro nutricosmético muito conhecido é o colágeno, utilizado por $14 \%$ da população avaliada. O colágeno apresenta muitos benefícios para a pele, especialmente na melhora dos sinais de envelhecimento ${ }^{14}$. O colágeno é a maior classe de proteína fibrosa insolúvel encontrada na matriz extracelular e nos tecidos conjuntivos, cuja principal ação é de ordem estrutural ${ }^{15}$.

O ácido ascórbico ou vitamina $\mathrm{C}$, como é popularmente conhecida, participa de processos celulares de oxirredução, na biossíntese das catecolaminas e é 
importante na defesa do organismo contra infecções ${ }^{16}$. Além disso, é essencial para formação das fibras colágenas existentes nos tecidos do corpo humano, aumento da firmeza e elasticidade da pele, possui efeito clareador e é um poderoso antioxidante ${ }^{17}$. Do total da amostra deste estudo, 8 (8,6\%) indivíduos relataram o uso desse produto.

O nutricosmético de ácido graxo ômega-3 também tem sido usado para tratar várias afecções, dentre elas a dermatite atópica e psoríase ${ }^{9}$. Há evidências de efeitos anti-inflamatórios e estruturais do ácido graxo ômega 3 , associado ao ácido $\gamma$ linolênico $(G L A)^{18}$. Ômega 3 age na epiderme da pele humana, inibindo a síntese de moléculas pró-inflamatórias, além de desempenhar um papel estrutural físico na integridade da barreira da pele ${ }^{9}$. Já foi demonstrada significativa melhora na pele em relação ao tratamento de acnes ${ }^{9}$. Neste estudo $9,7 \%$ indivíduos fizeram uso do nutricosmético de ômega 3.

$\mathrm{Na}$ amostra atual, $52,18 \%$ possuiam indicação de algum profissional da saúde. Os demais obtiveram informações de familiares, amigos, propagandas. Sabe-se da importância da indicação correta de nutricosméticos, já que a automedicação pode levar a reações adversas, sendo fundamental o acompanhamento de um profissional da saúde para a preservação da saúde do indivíduo.

Este estudo apresenta algumas limitações. Primeiro, o delineamento é transversal, o que impossibilita averiguar a relação de causa e efeito. Segundo, o preenchimento incompleto dos questionários que impactaram no tamanho amostral.

\section{CONCLUSÃO}

O perfil de pacientes que frequentam os laboratórios de Estética e Cosmética da Universidade de Caxias do Sul quanto à utilização de nutricosméticos são principalmente mulheres de média idade, que utilizam com mais frequência o nutricosmético de vitamina $\mathrm{D}$.

Nota-se a importância de divulgações e pesquisas sobre nutricosméticos, já que o mesmo não está relacionado apenas a estética, mas sim a prevenção de disfunções sistêmicas do organismo e a manutenção da saúde. Mais estudos são necessários para elucidar a associação entre o uso de nutricosméticos e variáveis de estilo de vida, utilizando maiores tamanhos amostrais. 


\section{REFERÊNCIAS}

1 Silva CN, Almeida FQ, Gomes IM. Beleza e feminilidade: O corpo feminino. Revista Vida Capichaba (1925-1939) 2015 Nov; 27(46):35-52.

2 Berger M. Corpo e identidade feminina. São Paulo. Tese [Doutorado em Antropologia] -Universidade de São Paulo; 2006.

3 Nunes MD. Produtos Cosméticos como Objetos De Fronteira. Florianópolis. Tese [Pós-Graduação em Sociologia Política]- Universidade Federal de Santa Catarina; 2016.

4 Ruiz BFN, Lonni A, Carreira C, Ruiz K, Palma G, Gonçalves V. Nutricosméticos: um conceito inovador. Visão Acadêmica 2014 abr./jun.;15(2):106-128.

5 Cavichioli B, Abourihan, CLS, Passoni CMS. Monitoramento da administração de um suplemento como coadjuvante na perda de peso. Cad. da. Esc.de Saúde 2012; 1(7):90-110.

6 Anunciato T, Rocha Filho PA. Carotenoids and polyphenols in nutricosmetics, nutraceuticals, and cosmeceuticals. Journal Of Cosmetic Dermatology. 2012 Fev;11(1):51-54.

7 Bittencourt LJ. Padrões de beleza e transtornos do comportamento alimentar em mulheres negras de Salvador / Bahia. Bahia. Tese [Pós Graduação do Instituto de Saúde Coletiva (ISC)] - Universidade Federal da Bahia; 2013.

8 Berger M. Corpo e identidade feminina. São Paulo. Tese [Doutorado em Antropologia]- Universidade de São Paulo; 2006.

9 Jung J, Kwon H H, Hong J S, Yoon J Y, Park M S, Jang M Y et al. Effect of Dietary Supplementation with Omega-3 Fatty Acid and Gammalinolenic Acid on Acne Vulgaris: A Randomised, Double-blind, Controlled Trial. Acta Dermato Venereologica. 2014; 94(5):521-525.

10 Proksch E, Schunck M, Zague V, Segger D, Degwert J, Oesser S. Oral Intake of Specific Bioactive Collagen Peptides Reduces Skin Wrinkles and Increases Dermal Matrix Synthesis. Skin Pharmacology And Physiology. 2014;27(3):113119.

11 Marques CDL, Dantas AT, Fragoso TS, Duarte ALBP. A importância dos níveis de vitamina D nas doenças autoimunes. Rev Bras Reumatol. 2010;50(1):67-80.

12 Striegel $H$, Simon $P$, Wurster $C$, Niess A M, Ulrich R. The Use of Nutritional Supplements Among Master Athletes. International Journal Of Sports Medicine. 2005 Jul;27(3):236-241.

13 Holick MF. Vitamin D deficiency. New Engl J Med. 2007;357:266-8. 
14 Zague V, Machado-Santelli GM. Bases Científicas Dos Efeitos da Suplementação Oral com Colágeno Hidrolisado na Pele. Revista Brasileira de Nutrição Funcional. 2016; 5(65):19-25.

15 Porfirio E, Fanaro GB. Suplementação com colágeno como terapia complementar na prevenção e tratamento de osteoporose e osteoartrite: uma revisão sistemática. Rev. bras. geriatr. Gerontol [Acesso em: 27 out. 2019]; 2016 Feb;19(1):153-164. Disponível em: http://www.scielo.br/scielo.php?script= sci_arttext\&pid=S180998232016000100153\&lng=en\&nrm=iso.

16 Azulay MM, Mandarim-de-Lacerda CA, Perez MA, Filgueira AL, Cuzzi T. Educação Médica Continuada Vitamina C. An bras Dermatol; 2003 maio/jun.;78(3):265-274.

17 Santos MP, Oliveira NRF. Ação das vitaminas e antioxidantes na prevenção do envelhecimento cutâneo. Disciplinarum Scientia. Série: Ciências da Saúde; 2014; 15(1):75-89.

18 Menendez JA, Lupu R, Colomer R. Exogenous supplementation with omega-3 polyunsaturated fatty acid docosahexaenoic acid (DHA; $22: 6 n-3)$ synergistically enhances taxane cytotoxicity and downregulates Her-2/neu (c-erbB-2) oncogene expression in human breast cancer cells. Eur J Cancer Prev. 2005; 14: 263-270.

19 Pérez EB. Revisión de los efectos beneficiosos de la ingesta de colágeno. Nutricion Hospitalaria; 2015 Jul;(1):62-66.

20 Dini I, Laneri S. Nutricosmetics: A brief overview. Phytotherapy Research. 2019 Set; 1(10):284

21 American Society of Plastic Surgeons [ASPS] [Acesso: 13. Nov. 2019]. Disponível em: http://www.isaps.org

22 Coelho FD, Carvalho PHB, Fortes LS, Paes ST, Ferreira MEC. Body dissatisfaction and influence of media in women who undergo plastic surgery. Revista Brasileira de Cirurgia Plástica (RBCP): Brazilian Journal Of Plastic Sugery. 2015;30(4):567-573

23 BRASIL, Portal do Planalto. Presidência da República. Lei nº 9.782, de 26 de janeiro de 1999. [Acesso em: 19 maio 2019] Disponível em: http://www.planalto.gov.br/ccivil_03/leis//9782.htm.

24 BRASIL, Portal do Planalto. Presidência da República. Decreto no 3.571, de 21 de agosto de 2000. [Acesso em: 19 maio 2019] Disponível em: http://www.planalto.gov.br/ccivil_03/decreto/D3571.htm.

Artigo recebido em: 09/12/2019

Artigo aprovado em: 22/06/2020

Artigo publicado em: 06/07/2020 